\title{
Téoros
}

Revue de recherche en tourisme

\section{Les agences de voyage vont-elles finir dans le fossé des inforoutes?}

\section{Philippe Le Roux et François Bédard}

Volume 14, numéro 3, automne 1995

Tourisme et technologies de l'information

URI : https://id.erudit.org/iderudit/1075079ar

DOI : https://doi.org/10.7202/1075079ar

Aller au sommaire du numéro

Éditeur(s)

Université du Québec à Montréal

ISSN

0712-8657 (imprimé)

1923-2705 (numérique)

Découvrir la revue

Citer cet article

Le Roux, P. \& Bédard, F. (1995). Les agences de voyage vont-elles finir dans le fossé des inforoutes ? Téoros, 14(3), 8-10. https://doi.org/10.7202/1075079ar d'utilisation que vous pouvez consulter en ligne. 


\section{LES AGENCES DE VOYAGE VONT-ELLES FINIR DANS LE FOSSÉ DES INFOROUTES? Philippe Le Roux et François Bédard}

\author{
Philippe Le Roux \\ est associé de V(DL) 2 lnc. \\ animateur du Comité de veille \\ sur les inforoutes, co-auteur \\ d'Internet Secrets \\ (e-mail: leroux@vdl2.ca) \\ François Bédard \\ est professeur au département \\ d'études urbaines et \\ touristiques, Université du \\ Québec à Montréal et \\ coordonnateur du présent \\ numéro de Téoros \\ le-mail: \\ bedard.françois@uqam.ca) \\ (http://www.uqam.ca)
}

\section{Mode ou tendance?}

Depuls quelques années, mals surtout an cours des douze dernlers mols, les vocables "nouvelles technologles", "mult/médla", "autoroute électronlque" et "Internet" sont rếpétés à satlété par les médlas, les pollticlens et par les anaIystes dans presque tous les domalnes d'activités économlques. Certalns prédisent des paradls virtuels, à défaut d'artuflclels, tandls que d'autres nous alertent sur l'enfer que représentent ces technologles. Dans ce broulıalıa contradictolre, chaque cltoyen, chaque professlonnel se retrouve un peu seul pour falre le polnt face aux défls que représente cette convergence numérlque des technologles.

Il est utlle de distinguer ce qul est du domalne de la mode de ce quil s'inscrit dans une tendance lourde. Ce qul est du domane de la mode va prochalnement rejolndre dans les oubllettes technologlques les déclarations de ceux qul annonçalent, depuls plusleurs années délà. la dlsparıtıon du papler dans les bureaux, la disparition du cinéma et des journaux ou la disparition des voyages daffaires à cause de la vidéoconférence. Par contre, les tendances lourdes se caractérisent par des phénomènes qul sont en tralı de s'Implanter et qui modifieront profondément notre avenir. Par exemple, la réalité donne ralson à ceux quil annonçalent depuls plusleurs années que les réseaux télématlques, les applications Interactives ou les inforoutes allalent s'implanter et changer notre facon de falre. En effet, on observe que les affalres se font de plus en plus sur ces réseaux. notamment sur Internet. Par contre. ceux qul pensalent que telle ou telle nouvelle technologle fermée, comme UBI, allalt remplacer tout ca, ont confondu mode et tendance lourde, et. sont en traln de déchanter rapidement face à ce quil, dans les prochalns mols, va apparaître comme un échec aussl net. qu'Alex(1) l'a été en 1990 à molns d'une refonte totale du projet et de ses principes de base.

\section{Menace ou opportunité?}

De nombreuses professlons sont en tualn d'être complètement bouleversées, volre totalement supprimées par lapparltion de nouvelles technologles. Plisleurs professlons. comme les typographes et les dessinateurs traditionnels, ont disparu ou sont devenues marginales, tandls que d'autres commencent à connaître de grandes difflcultés, comme les
Ingénleurs qui, au Québec ont un fort taux de chômage. Qu'en est-Il pour les agents de voyages?

Les agences de voyage n'échappent pas à l'environnement technologlque. Le phénomène n'est pas nouveau. En regardant le passé, II y a lieu d'être optimlste sur la capacité d'adaptation des agents de voyages a l'environnement technologlque. En effet, les agents de voyages ont su s'adapter aux nouvelles technologles et en turer profit. Ils ont été parml les premlers professlonnelş à utillser des réseaux télématlques (les prémlsses des inforontes) avec les multuples systèmes. Interactifs de réservation qu'lls utilisent. quotidlennement depuis plusleurs années. Ce fait est Important car II fournit des réponses à certaines des questlons fondamentales.

Les Inforoutes vont-elles s'implanter dans l'industrle du voyage? Oul, elles y sont défà.

Les agents de voyages sont lls capables d'intégrer les nouvelles technologles? Oul, Its en ont falt la preuve avant la plupart des autres professlons.

Ces technologles sont-elles stratéglques pour l'industrle du voyage? Oul. Il n'y a qu'à se rappeler pour s'en convalncre la batallle entre Alr Canada et Canadlen autour dı système de réservalton Gémlull.

Les technologles qul sont délà blen Implantées peuvent-elles rendre lypothétlque le succes des nouvelles? On n'a Jamals pu artêter le changement dans l'histolre de l'humanté avec tout ce qu'll comporte d'avantages et d'inconvénlents.

Oul. à part les technologues, les vendeurs dilıformatlque et les entreprises de télécommunications, ont Intérit à volr les nouvelles technologles de l'information se développer? Pourquol est-on sl sî́r quatelles vont contlnuer à s'implanter dans l'industrle du voyage? Tout simplement parce que c'est l'Industrle du voyage elle-même qul les réclame ces technologles.

Lindustrle du voyage a détà sufflsamment Intégré les technologies existantes pour qu'on envisage l'avenir immédiat davantage dans une ếvolutlon accélérée plutôt qu'une rếvolutloil.

\section{"Techuology push ou market pull?"}

Lapparition des systèmes de réservation informatisés n'est pas le falt de la disponibllité des réseaux mals plutôt la réponse à un besoln. De ınêne, ce ne sont pas les annonces des compagnles de téléplione ou des fabricants d'ondinateur quul poussent les fournlsseurs à voulour translger dlirectement. avec les cllents par dessus les agents de voyage; cest plutôt 
la tendance lourde observée, depuls quelques années, chez les principales compagnles de l'Industrle du voyage à se restructurer dans une concentration tant horizontale que verticale.

Les technologles ne sont que les outlls que tels et tels joueurs utllisent pour mettre en oeuvre leurs stratégles. Alnsl, ce qui peut constituer une menace pour les agences de voyages, ce n'est pas que les consommateurs pulssent faire une réservation depuls leur salon, mals plutôt que les fournlsseurs et les grossistes solent prêts à leur fournlr directement le service de réservation. Tout se déroule dans un contexte où les technologles et les stratégles Industrlelles s'allmentent mutuellement. Ce sera la responsabilité des historiens de demaln de trancher qui de la poule - les tendances Industrlelles, ou de l'oeuf - la technologle, a commencé le cycle,

\section{Tendances du marché et de l'industrie}

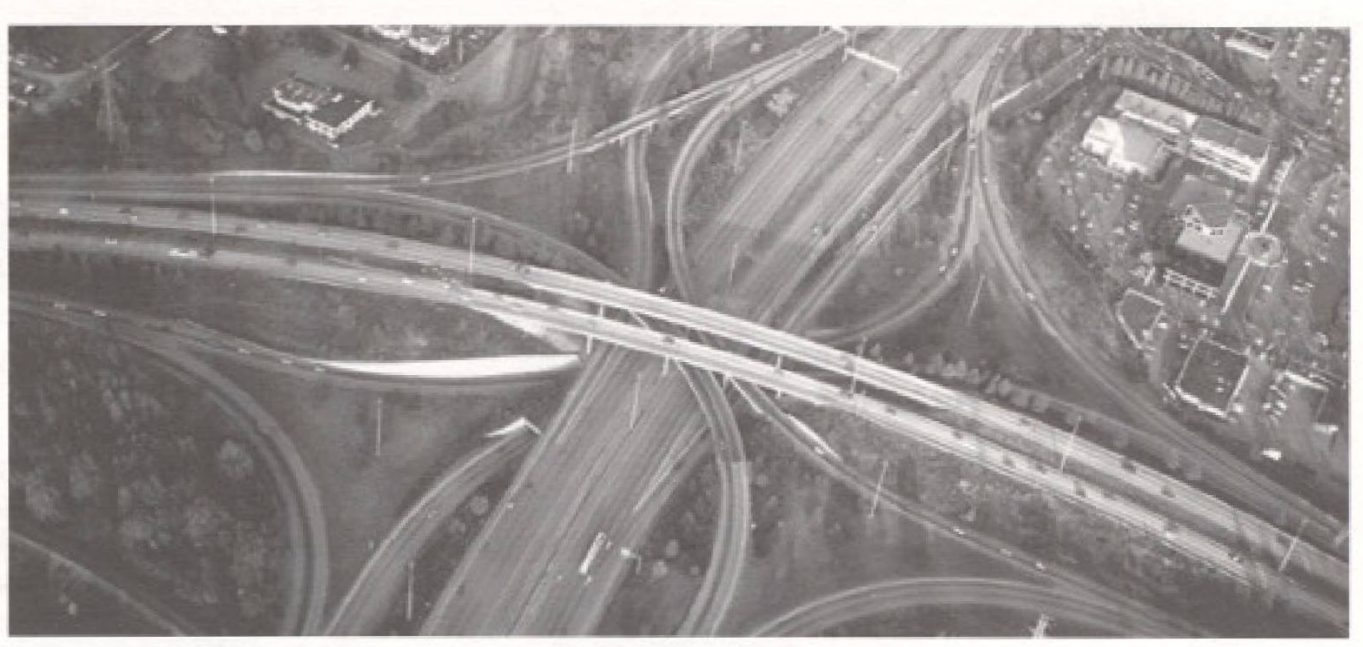

succès. II est probable que cecl accélère la spéclallsation des agences de voyage. Pendant que certalnes vont se posithonner encore plus fortement sur le prix, d'autres vont se spéclallser sur le voyage d'aventure ou le voyage corporatuf. Celles quil n'ont pas d'autre spécificlté que leur locallsatıon ne pourront plus compter longtemps sur cet élément de différenclation. En effet, déjà aujourd'hut, les clients qul réservent des voyages sur les systèmes d'America OnLine, d'Internet ou d'Amerlcan Express ne se demandent jamals où est situé leur fournisseur. Même la protection du permis d'a-
Comment les agences de voyages dolvent-elles se positjonner dans un contexte d'évolutlon technologique rapide? La mellleure façon de ne pas falie d'erreurs stratéglques - celles-cl peuvent être fatales consiste à revenir à l'élément le plus fondamental de l'entreprise. Cet élément, ce ne sont pas les partitions orchestrées par les grands Joueurs de l'industrle, pas plus que les annonces des promoteurs technologlques. c'est plutôt la mlsslon ou la ralson d'être de l'agence de voyage : satısfaire ses cllents.

Les utillsateurs et non les fournisseurs sont la composante de tous les projets d'inforoute quil déterminera lesquels seront des succès et lesquels échoueront. Les motivations des consommateurs ne sont pas diffilles à Identifler. IIs velllent le melleur servlce, la plus grande personnallsation et le melleur cô̂t. Certalns agents pourralent réagir en disant que les cllents sont Insensés à voulolr en même temps plus de services à molndre con̂ts. Mals, qu'on les trouve sensé ou pas, là n'est pas le problème. L'important c'est. de se posltJonner en fonction de ce que les cllents veulent.

Les technologles peuvent permettre de rédulre les coûts d'opération, de rejolndre un plus grand bassin de cllents, d'accroître la valeur aloutée du service ou de rédutre les délals et les intermédlaires. Il est rlsqué de courir après tous ces objectufs à la fols. Chaque agence devra chercher à se situer par rapport à ses concurrents, par rapports aux autres Intervenants de l'industrle (grossistes et fournlsseurs), par rapport aux marchés et par rapport aux évoluthons probables de sa profession. $\dot{A}$ partir de ce positionnement, elle devra se doter d"une stratégle d'affalres à moyen terme et identifler les technologtes qui peuvent l'alder à appliquer cette stratégle avec les melleures chances de gent de voyage déltvré par les autorités gouvernementales rlsque de subir des transformations sous pelne d'isoler totalement son Industrle du tourlsme du commerce mondlal.

\section{Quelles technologies pour quelles agences?}

Les agences qui veulent se positlonner sur le prix dolvent étudler quelles technologles elles peuvent mettre en place pour réduire leurs coûts dl'opération et de vente. Lutullsation de catalogues électroniques et de systèmes électronlques de commande peut être un bon moyen. Un catalogue électronIque sur Internet coûte molns cher à réallser dès la premlère année que la verslon papler et représente peu de frals pour les mises à jour subséquentes.

Les agences quil vont se spéclalliser dans des types de voyage précls, vont devolr probablement investiguer d'autres technologles, notamment celles qul vont leur permettre de rechercher des Informations polntues pour répondre aux besolns spéciflques de leurs cllentèles, alnsi que celles qul donneront à leurs cllents les outuls nécessalres pour y nccéder. C'est. là que la mise en place de banques d'informathou multimédia sur Internet, ou sur un CD-Rom pour le cllent pourra jouer un rồle. De la même façon la maîtrise des outuls technologlques de recherche d'information par l'agent de voyage lui permettra de trouver les informations pertinentes pour répondre aux demandes plus pointues de ses cllents.

\section{L'agence de voyage est-elle un fournisseur ou un utilisateur sur les inforoutes?}

Lagence de voyage devra probablement joner à la fols le rôle de fournisseur et celul d'utllisateur sur les inforoutes. 
Lagent devra être capable de produlre des contenus et des applications quI répondront aux attentes de ses cllents. comme la mlse à disposition d'un catalogue et d'un moyen de commande télématique et, surtout, de l'informatlon que peut. voulolr le consommateur avant de prendre sa déclsıon. II devra, à travers ces contenus et ces applications, dépasser son rôle de simple Intermédlalre entre le grosslste et le cllent. II pourra éventuellement mettre son client en contact avec d’autres qui ont dé|à expérimenté le service ou la destlnation proposés donnant alnsi accès à une evaluatlon supplémentalre à celle provenant du grossiste ou de l'agent. de voyage lul-même.

L'agence de voyage devra, et devralt délà, utillser les inforoutes pour trouver l'information que soulialte le cllent. Comme la démonstration en a été falte l'année derulère au congrès de l'ACTA sur le bateau M.V. Royal Majesty, quelques minutes de recherche ont permis de réunlr plus d'informatlon sur la crolslềre quaucun agent n’en avalt obtenu par d'autres moyens. Internet permet délà d'accéder à des dlzalnes de milliers de banques d'Informatlon sur tous les sujets et de rejolndre des gens de tous les domalnes partout dans le monde. Des dizalnes de CD-Rom existent et devralent être utulsés par les agents de voyage pour rechercher l'information qu'attendent leurs cllents plutôt que d'être dépendants de la disponibulté et de la connalssance de leur grossiste.

La question n'est pas de savolr si l'on est pour ou contre ces technologles et ces inforoutes, pas plis que de savoli si l'on dolt être ou ne pas être un usager, mals beaucoup plus de savolr quelles technologles et inforoutes utillser, à quelles flns et, surtout, dans le cadre de quelle stratégle de développement. De nombreuses entreprlses, dans le domalne du voyage comme alleurs, sont sollicltées pour ouvrlir une vitrine sur Internet ou partlclper àl'élaboratlon d'un CD-Rom. SI on falt une analogle entre la techuologle et les médlcaments. I'Important n'est pas d'en prendre. mals plutòt. de savolr lesquels prendre, quand, comblen et pourquol? Lagent de voyage devralt en priorite consacrer ses energles et ses moyens à définir sa stratégle et à identifier quelle technologle et quels médlas peuvent lul permettre de réussir. Les entreprlses qul ont apprls à utıllser stratéglquement l'Information dlsponible sur Internet ont. en moyenne, gagné beaucoup plus que celles qul se sont dépếchés de s"lnstaller dans un centre commerclal électronlque; et ce à blen melleur contt.

\section{Ouelques définitions}

Inforoute: On appelle Inforoute, un projet de réseau qui permet de transporter des Informations numériques incluant du texte, des données, du son et des Images. Les princlpes fondamentaux d'une inforoute sont la possibilité de communlquer directement entre les usagers, la possibilité pour chaque cllent d'être autant un producteur qu'un consommateur d'Information et l'ouverture aux autres Inforoutes. On peut clter Internet, les réseaux télématlques, Sabre, etc.

Internet : Vaste réseau Interconnectant plusleurs dlzalnes de milllers de réseaux à travers le monde. Créé en 1969. Internet est, devenu accessible à tous depuis près de quatre ans. Les applicatilons princlpales d'lnternet sont le courrler électronlque (plus de $60 \mathrm{mllllons}$ de personnes à travers le monde). les systèmes de communlcation de groupe (Newsgroups, débats par courrier électronlque, listes d'envol) et le World Wide Web, ensemble de millions de pages d'information multumédia produltes tant par des instututions que par des Indlvidus et qul sont toutes rellées les unes aux autres

par des llens hypertexte. Internet quil connaît un taux de crolssance de 8 à $10 \%$ par mols, est aujourdhul considérée comme le troncon central de toutes les autres inforoules.

UBI : Prolet de réseau fermé ou des fournlsseurs peuvent mettre de I'Information et des applications Interactives à la disposition d'utilisateurs. Basé sur le réseau du câble, la télévislon et un décodeur Informatlque, UBI n'est pas à proprement parler une Inforoute. Les récentes annonces d'Hydro-0uébec conjuguées aux retards et défectlons parml les partenalres d'UBI lalssent penser qu'UBI ne sera blentôt plus qu'une application parml d'autres sur l'Inforoute que compte développer Hydro-Québec dans son projet Hydro-Québécom.

SIRIUS : Projet d'infrastructure technologlque de 8 mIlliards de dollars annoncé par Stentor, consortjum des compagnies de téléphone du Canada. Stentor n"est pas à proprement parler une liforoute, mals beaucoup plus un projet de inodernlsatlon des réseanx de communlcations des compagnles de téléphone aftn de leur permettre de rencontrer les besolns teclinologlques que vont créer les Inforoutes dans les années à venlir.

CD Rom : DIsque Optlque permettant de stocker un volume Important d'Informatlons numérlques et pouvant être consultées sur un mlcro-ordinateur doté du lecteur approprié. Le CD-Rom n'est pas une Inforoute, pas plus qu'une applicatlon. II est. un support très adapté au multimédla et quI tend de plus en plus à se comblner aux Inforoutes. Le CD-Rom est un médilum Idéal pour stocker et dlstribuer plyzslquement. des gros volumes d'information statlque. II a pour défaut de ne pouvolr être mis à jour.

Réseaux télématiques : Réseaux d'Informatlon, de communlcation Interpersonnelle et de transactlons tarlfées à l'utillsatilon. Leur principale caractéristique est que lopérateur foue un rôle de diffuseur et de factureur des contenus établis Indépendamment par les fournlsseurs de service. Prédécesseurs des Inforoutes, Ils leur ont ouvert le chemin. Leur principale IImilte a tralt à leur non-unlversalltế et à leur coût d'utllisatlon. Par exemple, à Montréal. Internet revlent à molns de is de lheure alors quAmerica OnLine conte plus de 10 \$ de l'heure, Regroupant plus de dix millions d'utilisateurs, ces réseaux ont dû se reller à Internet pour ne pas disparaître. On peut citer par exemple Amerlca OnLine, Compuserve. Prodlgy. Genle. Microsoft Nelwork et. le minltel.

Notes

(1) Alex est un projet télématique lancé par Bell en décembre $1988 \bar{s}$ quil a atteint

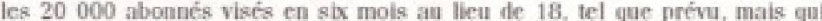
sest vite etiole. notamment hanse dune tarifical ion abnshe et diun trop gros contrile du contenu par Bell Canada. 